\title{
STATISTICAL ANALYSIS OF SURVIVAL TIMES BASED ON PROPORTIONAL GENERALIZED ODDS MODELS
}

\author{
Xiaohu Li, Linxiong $\mathrm{Li}^{*}$, Rui Fang ${ }^{\dagger}$ \\ Department of Mathematical Sciences \\ Department of Mathematics \\ School of Mathematical Sciences
}

\begin{abstract}
In the area of survival analysis the most popular regression model is the Cox proportional hazards $(\mathrm{PH})$ model. Unfortunately, in practice not all data sets satisfy the PH condition and thus the PH model cannot be used. To overcome the problem, the proportional odds (PO) model ( Pettitt 1982 and Bennett 1983a) and the generalized proportional odds (GPO) model ( Dabrowska and Doksum, 1988) were proposed, which can be considered in some sense generalizations of the $\mathrm{PH}$ model. However, there are examples indicating that the use of the PO or GPO model is not appropriate. As a consequence, a more general model must be considered. In this paper, a new model, called the proportional generalized odds (PGO) model, is introduced, which covers PO and GPO models as special cases. Estimation of the regression parameters as well as the underlying survival function of the GPO model is discussed. An application of the model to a data set is presented.
\end{abstract}

Key words: Burr distribution; Frailty parameter; Maximum likelihood estimation; Censored data.

\section{Introduction}

When lifetime is subject to right censoring one of the commonly used regression models is the Cox proportional hazards $(\mathrm{PH})$ model, provided that the data satisfies certain $\mathrm{PH}$ conditions(Cox 1972). Since it is not uncommon that the PH conditions are violated, different regression models need to be constructed to fit the data. One of them is the proportional odds (PO) model proposed by Pettitt (1982) and Bennett (1983a, b). Just like the PH model, the PO model assumes that the odds, instead of the hazard rates, of the distribution of a lifetime are proportional to the odds of a baseline distribution. It should be mentioned that the PO model is not a simple generalization of the PH model. Studies on the PO model can be found in Pettitt (1984), Bickel (1986), Cheng et al (1995), Cuzik (1988), Dabrowska and Doksum (1988), Shen (1995), Wu (1995), and Murphy et al (1997) among others. Most of the studies mainly focus on

*The corresponding author. E-mail: 1li1@uno.edu.

${ }^{\dagger}$ Supported by National Natural Science Foundation of China (11171278). 
estimation of the underlying survival function. In addition, for two independent samples with right-censored lifetimes, Dauxois and Kirmani (2003) developed a method for testing whether the data satisfies the PO assumption.

Besides the PO model a generalization of it was proposed. For two-sample lifetime data, Dabrowska and Doksum (1988) introduced a model, called the generalized proportional odds (GPO) model, and developed a testing method for comparison of two survival functions satisfying the GPO condition. For more details, one refers to Clayton and Cuzick (1986), Dabrowska et al (1989), Banerjee et al (2007) and references therein.

Although the GPO model is quite general and flexible to fit data, there are still many cases where the application of the GPO model is not suitable (See Section 4). To this end, we propose a more general model, called proportional generalized odds (PGO) model. The main difference between the GPO model and the PGO model is that the former assumes a common frailty factor for two groups of survival times under study and the latter allows the two groups to have different frailty factors. As will be seen in Section 4, the GPO model does not fit the data, while the proposed PGO model fits. The major differences between Bennett (1983a) and the current study are that (i) the current PGO model is more general than the PO model and (ii) the current study includes regression models but Bennett (1983a) does not.

The rest of this paper is organized as follows: In Section 2, the PGO model is defined and discussions on the relation between it and other related models are presented. Section 3 discusses semi-parametric estimation of the PGO model. In Section 4, the PGO model is applied to a data set about kidney disease recurrent times. Some discussions are presented in Section 5.

\section{Proportional generalized odds model}

Let $T \geq 0$ denote a lifetime and $\mathrm{z}$ a vector of covariates. Assume that the survival function $S(t)=\mathrm{P}(T>t)$ of $\mathrm{T}$ is continuous. The ratio $[1-S(t)] / S(t)$ is called odds, which measures the odds of "failure" against "survival" at time $t \geq 0$. Pettitt (1982) and Bennett (1983a, b) introduced the PO model as follows,

$$
\frac{1-S(t)}{S(t)}=\theta(z) \frac{1-S_{0}(t)}{S_{0}(t)}, \text { for all } t \geq 0,
$$

in which $S_{0}$ is a baseline survival function. This model assumes that lifetime $T$ is associated with its covariate $z$ only through $\theta$, which is independent of time $t$. This model actually assumes that the odds ratio (against a baseline) at any time $t$ is a constant $\theta$, free of $t$. This model has been applied to various data sets where the use of the PH model is not appropriate. As mentioned in Section 1, the PO model is a different model, not an extension of the PH model. In particular, if we let $\lambda(t)$ denote the hazard function corresponding to $S(t)$ then, under the PO model, the hazard ratio

$$
\frac{\lambda(t)}{\lambda_{0}(t)}=\left[1+(\theta(z)-1) S_{0}(t)\right]^{-1},
$$


which it is not a constant unless $\theta(z)=1$.

A more general model was introduced by Dabrowska and Doksum (1988) as follows. First, they extended the odds to generalized odds defined as

$$
r \frac{1-S^{1 / r}(t)}{S^{1 / r}(t)}
$$

Obviously, it reduces to the usual odds when $r=1$. Then they proposed the following GPO model

$$
\frac{1-S_{2}^{1 / r}(t)}{S_{2}^{1 / r}(t)}=\theta \frac{1-S_{1}^{1 / r}(t)}{S_{1}^{1 / r}(t)},
$$

where $S_{1}$ and $S_{2}$ are, respectively, survival functions of two independent samples, and $r$ is a (frailty) parameter. Since it is shown that the generalized odds converge to the cumulative hazard - $\log$ $S(t)$ as $r \rightarrow \infty$, the GPO model becomes the PH model as $r \rightarrow \infty$. That is, the GPO model covers the PH and PO models as special cases. Studies of the GPO model focus on testing for the hypothesis of equality of $S_{1}$ and $S_{2}$ when they satisfy the GPO condition, and the model has been studied extensively in the past two decades. Recently, we tried to apply the model to a data but found that the data does not satisfy the GPO assumption. For this reason, we extend the GPO model by allowing different $r$ for different sample. In particular, we propose the following proportional generalized odds (PGO) model,

$$
\frac{1-S^{1 / r}(t)}{S^{1 / r}(t)}=\theta \frac{1-S_{0}(t)}{S_{0}(t)}, \quad \text { for all } t \geq 0
$$

where $S_{0}$ denotes a baseline survival function, $r>0$ and $\theta>0$ are parameters. To compare it with the GPO model, let us consider two independent samples. Let $S_{i}$ denote the survival function of sample $i, i=1,2$. Then it is seen that

$$
\frac{1-S_{1}^{1 / r_{1}}(t)}{S_{1}^{1 / r_{1}}(t)}=\frac{\theta_{1}}{\theta_{2}} \frac{1-S_{2}^{1 / r_{2}}(t)}{S_{2}^{1 / r_{2}}(t)}, \quad \text { for all } t \geq 0
$$

So the PGO model is a generalization of the GPO model. It can be verified that Burr distribution (Burr, 1942) with different scale parameters (See Section 3 below) satisfies the PGO condition but not the PO condition.

In addition to the natural extension, the PGO model can also be obtained from an engineering point of view. Let $X_{1}, \cdots, X_{n}, \cdots$ be a random sample of observations with distribution function $F$ and survival function $S=1-F$, and let $N$ be a random variable having a geometric distribution with probability of "success" $\theta \in(0,1)$. Then Kirmani and Gupta (2001) showed that the distribution of lifetime $Y=\min _{1 \leq i \leq N} X_{i}$ of a "series" system satisfies the PO equation 


$$
\theta \frac{1-S_{Y}(t)}{S_{Y}(t)}=\frac{1-S(t)}{S(t)}, \text { for all } t \geq 0
$$

and the distribution of the lifetime $\mathrm{Z}=\max _{1 \leq i \leq N} X_{i}$ of a "parallel" system satisfies the PO equation

$$
\theta \frac{1-S(t)}{S(t)}=\frac{1-S_{Z}(t)}{S_{Z}(t)}, \quad \text { for all } t \geq 0
$$

These two equations provide a physical support to the PO model. In a similar manner, the following is for the PGO model in (2.1).

Proposition 1 Suppose that $N$ follows a negative binomial distribution with parameters $(r, \theta)$. Then, for all $t \geq 0$,

$$
\begin{aligned}
\theta \frac{1-S_{Y}^{1 / r}(t)}{S_{Y}^{1 / r}(t)} & =\frac{1-S(t)}{S(t)}, \\
\frac{F_{Z}^{1 / r}(t)}{1-F_{Z}^{1 / r}(t)} & =\theta \frac{F(t)}{1-F(t)} .
\end{aligned}
$$

Proof For any $t$, the survival function

$$
\begin{aligned}
S_{Y}(t) & =P\left(\min _{1 \leq i \leq N} X_{i}>t\right) \\
& =\sum_{n=r}^{\infty} P\left(\min _{1 \leq i \leq n} X_{i}>t\right) \cdot P(N=n) \\
& =\sum_{n=r}^{\infty} S^{n}(t) \cdot\left(\begin{array}{l}
n-1 \\
r-1
\end{array}\right) \theta^{r}(1-\theta)^{n-r} \\
& =\theta^{r} S^{r}(t) \sum_{n=r}^{\infty}\left(\begin{array}{l}
n-1 \\
r-1
\end{array}\right)[(1-\theta) S(t)]^{n-r} \\
& =\frac{\theta^{r} S^{r}(t)}{[1-(1-\theta) S(t)]^{r}} \sum_{n=r}^{\infty}\left(\begin{array}{l}
n-1 \\
r-1
\end{array}\right)[1-(1-\theta) S(t)]^{r}[(1-\theta) S(t)]^{n-r} \\
& =\frac{\theta^{r} S^{r}(t)}{[1-(1-\theta) S(t)]^{r}} .
\end{aligned}
$$

Thus, we have

$$
\frac{S_{Y}^{1 / r}(t)}{1-S_{Y}^{1 / r}(t)}=\frac{\theta S(t)}{1-(1-\theta) S(t)-\theta S(t)}=\theta \frac{S(t)}{1-S(t)} .
$$


Likewise, the distribution function

$$
F_{Z}(t)=\sum_{n=r}^{\infty} F^{n}(t) \cdot\left(\begin{array}{c}
n-1 \\
r-1
\end{array}\right) \theta^{r}(1-\theta)^{n-r}=\frac{\theta^{r} F^{r}(t)}{[1-(1-\theta) F(t)]^{r}},
$$

and thus

$$
\frac{F_{Z}^{1 / r}(t)}{1-F_{Z}^{1 / r}(t)}=\frac{\theta F(t)}{1-(1-\theta) F(t)-\theta F(t)}=\theta \frac{F(t)}{1-F(t)},
$$

for any $t$.

\section{Estimation}

A nonnegative random variable $X$ is said to follow a Burr distribution (Burr, 1942), denoted as $X \sim \operatorname{Bur}(\alpha, \sigma, r)$, if it has the survival function

$$
S(x)=\left(1+\frac{x^{\alpha}}{\sigma}\right)^{-r}, \quad x \geq 0,
$$

where the shape parameter $\alpha$, the scale parameter $\sigma$ and the frailty parameter $r$ are all positive. $\operatorname{Bur}(1,1, r)$ is called the standard Burr distribution.

Proposition 2 below shows that Burr distribution satisfies the PGO condition. Since it is straightforward, we state it without proof.

Proposition 2 Suppose $X_{i} \sim \operatorname{Bur}\left(\alpha, \sigma_{i}, r_{i}\right), i=1,2$. Then, for all $t \geq 0$,

$$
\frac{1-S_{2}^{\frac{1}{r_{2}}}(t)}{S_{2}^{\frac{1}{r_{2}}}(t)}=\theta \frac{1-S_{1}^{\frac{1}{r_{1}}}(t)}{S_{1}^{\frac{1}{r_{1}}}(t)}
$$

where $\theta=\sigma_{1} / \sigma_{2}>0$.

It is easy to verify that PGO equation (2.2) is invariant under positive increasing transformations. That is, if $X_{1}$ and $X_{2}$ satisfy (2.2), then so do $h\left(X_{1}\right)$ and $h\left(X_{2}\right)$ for any increasing function $h$. Proposition 3 below tells that any two random variables satisfying (2.2) may be regarded as increasing transformations of two Burr random variables with a common shape parameter.

Proposition 3 Suppose $X_{2}$ and $X_{1}$ satisfy the PGO model

$$
\frac{1-S_{2}^{\frac{1}{r_{2}}}(t)}{S_{2}^{\frac{1}{r_{2}}}(t)}=\theta \frac{1-S_{1}^{\frac{1}{r_{1}}}(t)}{S_{1}^{\frac{1}{r_{1}}}(t)}, \quad \text { for } \theta>0 \text { and all } t \geq 0 .
$$

Then, the transform 


$$
h(t)=\frac{1-S_{2}^{\frac{1}{r_{2}}}(t)}{S_{2}^{\frac{1}{r_{2}}}(t)}
$$

gives rise to $h\left(X_{2}\right) \sim \operatorname{Bur}\left(1,1, r_{2}\right)$ and $h\left(X_{1}\right) \sim \operatorname{Bur}\left(1, \theta, r_{1}\right)$.

Proof Note that $S_{2}\left(X_{2}\right)$ has the standard uniform distribution, we have, for any $t \geq 0$,

$$
P\left(h\left(X_{2}\right)>t\right)=P\left(\frac{1-S_{2}^{\frac{1}{r_{2}}}\left(X_{2}\right)}{S_{2}^{\frac{1}{r_{2}}}\left(X_{2}\right)}>t\right)=P\left(S_{2}\left(X_{2}\right) \leq(1+t)^{-r_{2}}\right)=(1+t)^{-r_{2}} .
$$

That is, $h\left(X_{2}\right) \sim \operatorname{Bur}\left(1,1, r_{2}\right)$, the standard Burr distribution. And in a similar manner it also holds that, for any $t \geq 0$,

$$
\begin{aligned}
P\left(h\left(X_{1}\right)>t\right) & =P\left(\frac{1-S_{2}^{\frac{1}{r_{2}}}\left(X_{1}\right)}{S_{2}^{\frac{1}{r_{2}}}\left(X_{1}\right)}>t\right) \\
& =P\left(\frac{1-S_{1}^{\frac{1}{r_{1}}}\left(X_{1}\right)}{S_{1}^{\frac{1}{r_{1}}}\left(X_{1}\right)}>\frac{t}{\theta}\right) \\
& =P\left(S_{1}\left(X_{1}\right) \leq\left(1+\frac{t}{\theta}\right)^{-r_{1}}\right) \\
& =\left(1+\frac{t}{\theta}\right)^{-r_{1}} .
\end{aligned}
$$

That is, $h\left(X_{l}\right) \sim \operatorname{Bur}\left(1, \theta, r_{l}\right)$.

We now discuss the PGO regression model. Assume that in model (2.1) survival function $S$ is affected by covariates $z$ only through $\theta$. Specifically, model (2.1) becomes

$$
\frac{1-S^{1 / r}(t)}{S^{1 / r}(t)}=\theta(z) \frac{1-S_{0}(t)}{S_{0}(t)}, \quad \text { for all } t \geq 0,
$$

where $\theta(z)=\exp \left\{\boldsymbol{\beta}^{\prime} z\right\}$

$$
z=\left(\begin{array}{c}
z_{1} \\
\vdots \\
z_{p}
\end{array}\right), \quad \boldsymbol{\beta}=\left(\begin{array}{c}
\beta_{1} \\
\vdots \\
\beta_{p} .
\end{array}\right)
$$

Let $T_{1} \sim S_{1}$ and $T_{2} \sim S_{2}$ be two independent lifetimes satisfying the PGO model 


$$
\frac{1-S_{i}^{\frac{1}{r_{i}}}(t)}{S_{i}^{\frac{1}{r_{i}}}(t)}=\theta(z) \frac{1-S_{0}(t)}{S_{0}(t)}, \quad \text { for some } r_{i}>0 \text { and } i=1,2
$$

Apparently it is a semi-parametric model. We now use the likelihood principle to estimate the baseline $S_{0}$, parameters $\beta$ and $r_{i}$, and survival functions $S_{i}(t ; z), i=1,2$, based on censored data. This can be considered as a two-sample problem with covariates. The same method can be used for three or more samples. Define

$$
Y_{i}=\left[1-S_{0}\left(T_{i}\right)\right] / S_{0}\left(T_{i}\right), \quad i=1,2 .
$$

Then, by Proposition 3, $Y_{i}$ follows a Burr distribution with survival function

$$
Q_{i}(y ; z)=\frac{1}{(1+\theta(z) y)^{r_{i}}}, \quad y \geq 0
$$

Since both samples share the same baseline $S_{0}$, we combine two samples into one in order to construct a pooled estimate of $S_{0}$. For simplicity let to denote the distinct observed lifetimes from the combined sample, of which some are from sample 1 and the others are from sample 2. Further, we let $t_{j}^{c}, j=1,2, \ldots$ denote censored lifetimes from the combined sample. In addition, we use $y_{(i)}^{o}$ and $y_{j}^{c}$, respectively, to denote the corresponding transformed values of $t_{(i)}^{o}$ and $t_{j}^{c}$, . Note that although it is not clearly labeled (for simplicity purpose), it is clear to us as for which sample an observation belongs to. Still for simplicity, we are going to use $r$ for both $r_{l}$ and $r_{2}, Q$ for both $Q_{1}$ and $Q_{2}$ and the density function of $Q$

$$
q(t ; z)=\frac{r \theta(z)}{(1+\theta(z) y)^{r+1}}
$$

for $q_{1}$ and $q_{2}$, the density functions of $Q_{1}$ and $Q_{2}$. With these notations, the likelihood function in terms of $Y$ takes the form of

$$
\mathcal{L}\left(\boldsymbol{\theta}, r_{1}, r_{2} ; \boldsymbol{y}\right)=\prod_{i}\left\{\prod_{l \in \mathcal{O}_{i}}\left[q\left(y_{(i)}^{o} ; \boldsymbol{z}_{(l)}\right) \frac{\mathrm{d} y_{(i)}^{o}}{\mathrm{~d} t_{(i)}^{o}}\right]\right\} \cdot \prod_{j}\left[\prod_{l \in \mathcal{C}_{j}} Q\left(y_{j}^{c} ; \boldsymbol{z}_{(l)}\right)\right],
$$

where $O_{i}$ is the set of subscripts of observed lifetimes tied at $t_{(i)}, C_{j}$ is that of right-censored lifetimes in $\left[t_{(j)}, t_{(j+1)}\right)$, and the derivative is Jacobian due to the transformation. Notice that $y$ 's as a function of $S_{0}$ are also unknown parameters. To obtain the likelihood estimates of all parameters, following two approximations are used, which are similar to Bennett (1983a). First we replace the derivative by its approximation $\frac{y_{(j)}-y_{(j+1)}}{t_{(j)}-t_{(j+1)}}$; second, we replace $Q\left(y_{j}^{c}, z\right)$ by $Q\left(y_{(j)}^{\prime}, z\right)$ where $y_{(j)}^{\prime}$ is the largest observed lifetime before $y_{j}^{c}$ from the same sample. Then the above likelihood function is approximated by 


$$
\prod_{i}\left\{\prod_{l \in \mathcal{O}_{i}}\left[\frac{r \theta\left(z_{k}\right)}{\left(1+y_{(i)}^{o} \theta\left(z_{k}\right)\right)^{r+1}} \frac{y_{(i)}^{o}-y_{(i-1)}^{o}}{t_{(i)}^{o}-t_{(i-1)}^{o}}\right]\right\} \cdot \prod_{j}\left[\prod_{l \in \mathcal{C}_{j}} \frac{1}{\left(1+y_{(j)}^{\prime} \theta\left(z_{l}\right)\right)^{r}}\right] .
$$

Once the likelihood (3.4) is maximized with respect to parameters $\beta, r_{1}, r_{2}$, and $y_{(i)}^{o} i=1$, $2, \ldots, k$, the baseline survival function $S_{0}(t)$ can be estimated by

\section{An application}

$$
S_{0}\left(t_{(i)}\right)=\frac{1}{1+y_{(i)}^{o}}, \quad i=1,2, \cdots, k
$$

This section applies the proposed PGO model to a data set about kidney patients recurrent times.

The data in Table 1 contains 38 kidney patients' second recurrent times to infection at point of insertion of the catheter for kidney patients using portable dialysis equipment, the risk variables are age, sex and type of disease coded as $0=$ glomerulo nephritis $(\mathrm{GN}), 1=$ acute nephritis (AN), 2 = polycystic kidney disease (PKD), 3 = other; in the event column, 1 denotes that time to infection was observed and 0 denotes that it was censored. The original data containing the first two consecutive recurrent times and sex had been studied by McGilchrist and Aisbett (1991) using a frailty model and by McGilchrist (1993) and Lam (1994) using the proportional hazards model. They all found that "sex" was not significant.

As an illustration we divide the data into two groups: AN and PKD in one group and GN and Other in another group. To see whether the Cox's PH model, the PO model or the GPO model is suitable for the data, we ignore covariates "age" and "sex" and then for each of the groups we obtained the estimate of the survival function using three different methods: Kaplan- Meier (KM) estimate, the PO model, and the GPO model. If, for example, the PO model fits the data, then there will be some $\theta>0$ such that

$$
\frac{1-\hat{S}_{2}(t)}{\hat{S}_{2}(t)}=\theta \frac{1-\hat{S}_{1}(t)}{\hat{S}_{1}(t)}, \quad \text { for all } t \geq 0
$$


Table 1: Kidney patients' second recurrent times

\begin{tabular}{|c|c|c|c|c|c|}
\hline $\begin{array}{c}\text { patient } \\
\text { No. }\end{array}$ & recurrent time & event & $\operatorname{sex}$ & age & disease type \\
\hline 1 & 16 & 1 & 1 & 28 & 3 \\
\hline 2 & 13 & 0 & 2 & 48 & 0 \\
\hline 3 & 28 & 1 & 1 & 32 & 3 \\
\hline 4 & 318 & 1 & 2 & 31.5 & 3 \\
\hline 5 & 12 & 1 & 1 & 10 & 3 \\
\hline 6 & 245 & 1 & 2 & 16.5 & 3 \\
\hline 7 & 9 & 1 & 1 & 51 & 0 \\
\hline 8 & 30 & 1 & 2 & 55.5 & 0 \\
\hline 9 & 196 & 1 & 2 & 69 & 1 \\
\hline 10 & 154 & 1 & 1 & 51.5 & 0 \\
\hline 11 & 333 & 1 & 1 & 44 & 1 \\
\hline 12 & 8 & 0 & 2 & 34 & 3 \\
\hline 13 & 38 & 1 & 2 & 35 & 1 \\
\hline 14 & 70 & 0 & 2 & 42 & 1 \\
\hline 15 & 25 & 0 & 2 & 17 & 3 \\
\hline 16 & 4 & 0 & 1 & 60 & 1 \\
\hline 17 & 177 & 1 & 2 & 60 & 3 \\
\hline 18 & 114 & 1 & 2 & 43.5 & 3 \\
\hline 19 & 159 & 0 & 2 & 53 & 0 \\
\hline 20 & 108 & 0 & 2 & 44 & 3 \\
\hline 21 & 562 & 1 & 1 & 46.5 & 2 \\
\hline 22 & 24 & 0 & 2 & 30 & 3 \\
\hline 23 & 66 & 1 & 2 & 62.5 & 1 \\
\hline 24 & 46 & 0 & 2 & 42.5 & 1 \\
\hline 25 & 40 & 1 & 1 & 43 & 1 \\
\hline 26 & 201 & 1 & 2 & 57.5 & 1 \\
\hline 27 & 156 & 1 & 2 & 10 & 0 \\
\hline 28 & 30 & 1 & 2 & 52 & 1 \\
\hline 29 & 25 & 1 & 1 & 53 & 0 \\
\hline 30 & 26 & 1 & 2 & 54 & 0 \\
\hline 31 & 58 & 1 & 2 & 56 & 1 \\
\hline 32 & 43 & 1 & 2 & 50.5 & 1 \\
\hline 33 & 30 & 1 & 2 & 57 & 2 \\
\hline 34 & 5 & 0 & 2 & 44.5 & 0 \\
\hline 35 & 8 & 1 & 2 & 22 & 3 \\
\hline 36 & 16 & 0 & 2 & 42 & 3 \\
\hline 37 & 78 & 1 & 2 & 52 & 2 \\
\hline 38 & 8 & 0 & 1 & 60 & 2 \\
\hline
\end{tabular}




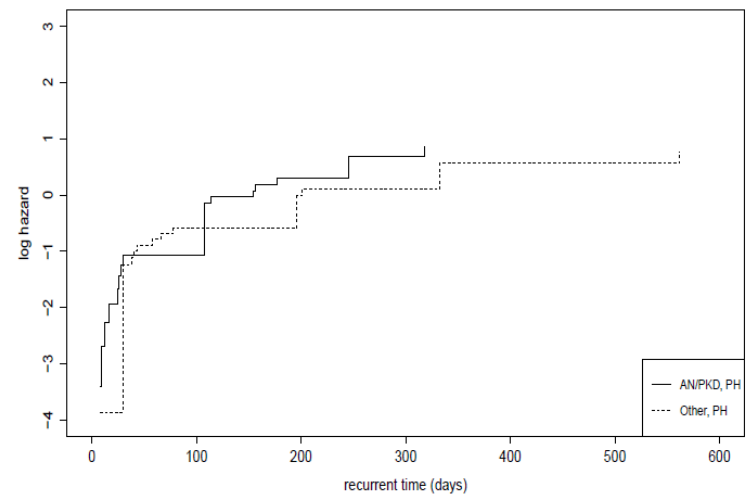

(a)Log hazard, PH model

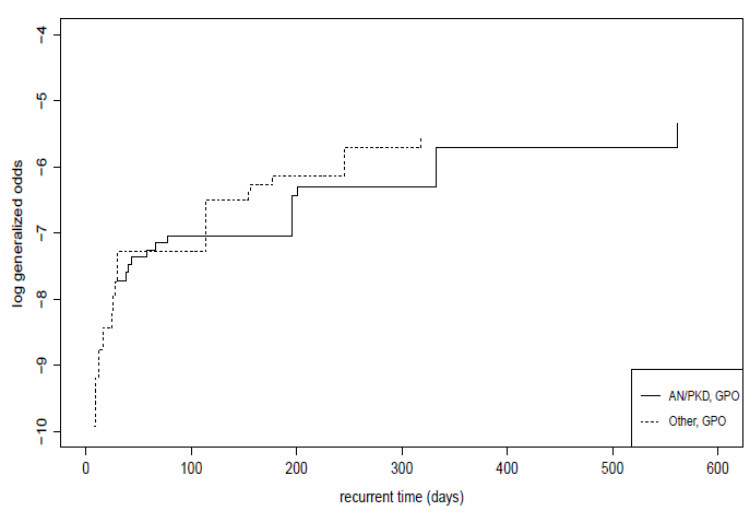

(c) Log generalized odds, GPO model

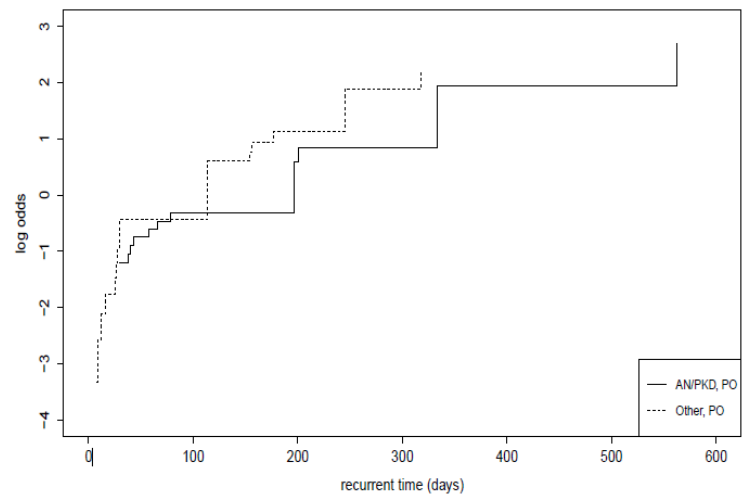

(b) Log odds, PO model

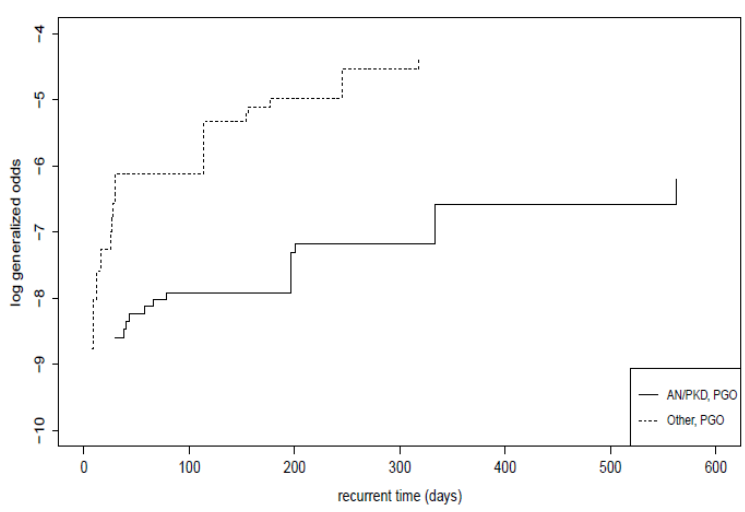

(d) Log generalized odds, PGO model

Figure 1: Log odds and log generalized odds

For the PH model, replace the numerator $1-S(t)$ in the above equation with its density function $f(t)$

. In other words, when taking log on both sides of the above equation, the two $\log$ (odds) curves (vs. time $t$ ) should be parallel if the model works. However, as is seen in Figure 1, the two $\log$ (hazard) curves based on the estimated hazard functions are not parallel (Fig 1(a)); the two $\log$ (odds) curves based on PO or GPO are not parallel either (Fig 1(b), 1(c)). By contrast,

the two curves based on the PGO model are nearly parallel (Fig 1(d)). This is evidence that the PGO model is more appropriate to fit this data than the other three models. In addition, goodness-of-fit tests for the hypothesis that the two groups (AN and PKD vs. GN and Other) satisfy the PO model using Neyman's smooth test and Kolmogorov-Smirnov test (see Kraus, 2009) have p-values of 0.014 and 0.094, respectively. This confirms our observation in Figure

1. By the way, if the data is divided into four groups, AN, PKD, GN, and Other, then the PH assumption is satisfied ( $p$-value $=0.503$ ); however, when the data is divided into two groups as 
described above, besides Figure 1(a), we performed a test and the result shows that the PH assumption is not satisfied ( $p$-value $=0.01329$ ), which is consistent with Figure 1(a).

We fit the PGO model to this data using likelihood (3.4). The genetic algorithm was employed for the computation. The maximum likelihood estimates of parameters $r 1, r 2$, and the coefficients of "age" and "sex" are, respectively, $\mathrm{e}^{5.5}, \mathrm{e}^{7.7}, 0.006$ and -1.29. A likelihood ratio test was performed for "age" and "sex" and found that none of them is significant ( $p$-value $=0.82$ for "age" and 0.17 for "sex").

\section{Discussions}

This paper proposes a model which generalizes existing PH, PO and GPO models. The example in Section 4 supports the proposed model. Although the proposed PGO model is more flexible than the existing models, it remains to be done to develop a method to verify whether a data satisfies the PGO condition. We believe that it is not an easy but a 'must' task. One of the most amazing part of the $\mathrm{PH}$ model is the partial likelihood estimate of the model parameters. Unfortunately, we are not able to construct a similar estimator for the proposed PGO model. So, large sample properties of our maximum likelihood estimators are not discussed. In addition, still because of the lack of partial likelihood function, estimations of the baseline survival function and other parameters are obtained in one step, instead of two steps like the PH model. This one step calculation involves heavier computation and more computer time. For our data in Section 4 Newton-Raphson algorithm was used first but it failed to converge; then we employed the genetic algorithm which worked very well. Without theoretical results, simulation studies are usually desired in order to investigate the performance of a proposed model. Unfortunately simulations are not done in this paper due to technical difficulties but we decided to conduct a thorough simulation study in the future. To summarize, this paper is a preliminary study of the problem; the above mentioned issues need to be studied in order to make the proposed model useful in practice.

\section{References}

[1] Banerjee, T., Chen, M., Dey, D. K. and Kim, S. (2007) Bayesian analysis of generalized odds-rate hazards models for survival data. Lifetime Data Analysis 13, 241-260.

[2] Bennett, S. (1983a) Analysis of survival data by the proportional odds model. Statistics in Medicine 2, 273-277.

[3] Bennett, S. (1983b) Log-logistic regression model for survival data. Applied Statistics 32, $165-171$

[4] Bickel, P. J. (1986) Efficient testing in a class of transformation models. Papers on Semiparametric Models at the ISI Centenary Session, Amsterdam, 63-81. 
[5] Burr, I. W. (1942) Cumulative frequency distributions. Annals of Mathematical Statistics $13,215-232$.

[6] Cheng, S. C., Wei, L. J. and Ying, Z. (1995) Analysis of transformation models with censored data. Biometrika 82, 835-845.

[7] Clayton, D. G. and Cuzick, J. (1985) The semi-parametric Pareto model for regression analysis of survival times. Bulletin of International Statististical lnstitute 51, 23.3:1-18.

[8] Cuzick, J. (1988) Rank regression. The Annals of Statistics 16, 1369-1389.

[9] Dabrowska, M. D. and Doksum, K. A. (1988) Estimation and testing in a two-sample generalized odds-rate model. Journal of American Statistical Association 83, 744-749.

[10]Dabrowska, M. D., Doksum, J. A. and Miura, R. (1989) Rank estimates in a class of semiparametric two-sample models. Annals of Institute in Statistical Mathematics 41, 63-79.

[11]Duxois, J. and Kirmani, S. N. U. A. (2003) Testing the proportional odds model under random censoring. Biometrika 90, 913-922.

[12]Kirmani, S. N. U. A. and Gupta, R. C. (2001) On the proportional odds model in survival analysis. Annals of the Institute of Statistical Mathematics 53, 203-216.

[13] Kraus, D. (2009) Checking proportional rates in the two-sample transformation model. Kybernetika 45, 261-278.

[14]Lam, K. F. (1994) Topics in Survival Analysis. PhD Thesis, the University of Hong Kong.

[15] McGilchrist, C. A. (1993) REML estimation for survival models with frailty. Biometrics 49, 221-225.

[16] McGilchrist, C. A. and Aisbett, C. W. (1991) Regression with frailty in survival analysis. Biometrics 47, 461-466.

[17]Murphy, S. A., Rossini, A. J. and Van der Vaart, A. W. (1997) Maximum likelihood estimation in the proportional odds model. Journal of American Statistical Association 92, 968-976.

[18]Pettitt, A. N. (1983) Inference for the linear model using a likelihood based on ranks. Journal of Royal Statistical Society 44, 234-243.

[19] Pettitt, A. N. (1984) Proportional odds models for survival data and estimates using ranks. Applied Statistics 33, 169-175. 
[20] Shen, X. (1998) Proportional odds regression and sieve maximum likelihood estimation. Biometrika 85, 165-177.

[21] Wu, C. O. (1995) Estimating the real parameter in a two-sample proportional odds model. The Annals of Statistics 63, 376-395.

Received November 19, 2014; accepted October 10, 2016.

Xiaohu Li

Department of Mathematical Sciences

Stevens Institute of Technology, Hoboken NJ 07030, USA

Linxiong Li*

Department of Mathematics

University of New Orleans, New Orleans, LA 70148, USA

Rui Fang ${ }^{\dagger}$

School of Mathematical Sciences

Xiamen University, Xiamen, Fujian 361005, China 
584 STATISTICAL ANALYSIS OF SURVIVAL TIMES BASED ON PROPORTIONAL GENERALIZED ODDS MODELS 